\title{
Solar radio bursts: Implications to the origin of in situ particles
}

Rositsa Miteva

\author{
Space Research and Technology Institute, Bulgarian Academy of Sciences, Sofia
}

E mail (rmiteva@space.bas.bg).

Accepted: 19 April 2018

\begin{abstract}
The significance of the solar radio burst signatures for the solar energetic particle (SEP) classification is investigated in the current report. The solar radio bursts associated with the proton-producing solar flares and coronal mass ejections are usually of types II, III and IV. Based on the solar radio burst occurrences in different wavelength ranges, several categories for the SEP origin are adopted. This study evaluates the statistical differences in the flare and CME event samples related to each of these proton categories: flare-driven, CME-driven and mixed-contribution.
\end{abstract}

(C) 2018 BBSCS RN SWS. All rights reserved

Keywords: solar energetic particles; solar flares; coronal mass ejections; solar radio bursts

\section{Introduction}

The use of remotely observed radio emission (produced by electrons) in the identification of the origin of solar energetic particles (SEPs: electrons, protons and heavy ions with $\mathrm{keV}-\mathrm{MeV}$ energies) is known for long time (e.g., Lin 1970). However, the same radio signatures (solar radio type II bursts) were used in the SEP classification proposed by Reames (1999). In the subsequent revisions, e.g., by Cliver (2009), Reames (2013) and the references therein, it was attempted to repair the initial inconsistency, namely the inclusion of type II radio burst in either category and prepare a more realistic SEP scheme.

Observations in radio wavelengths (from decimetric, dm, to kilometric, $\mathrm{km}$, range) provide an independent diagnostic tool for testing electron acceleration mechanisms and tracking the time evolution of the electron propagation, from the low corona to the Earth's orbit. Although comparing any electron signatures with in situ observed protons is an approximation, this indirect method is frequently used in the literature due to the availability of numerous proton catalogs compared to the lack of comprehensive electron lists to present date. Under the assumption that electrons and protons are accelerated by the same mechanism and in the same place, the radio emission will be used in the present study to relate to the origin of the in situ protons. This simplification is adopted in all studies that compare occurrence and parameters of in situ proton events with radio burst signatures generated by electrons. Case studies (Kallenrode and Wibberenz, 1991; Krucker and Lin, 2000) show that there are occasional differences in the proton and electron release times from the Sun based on estimations using various numerical techniques; however uncertainty ranges are not provided.

The radio emission is organized historically into several burst types (see reviews by Nindos et al. 2008, Pick and Vilmer 2008), depending on their appearance in the radio burst spectra, their time and frequency coverage, drift, etc., namely: type II (widely adopted as the signature of shock wave in the corona and/or interplanetary space), type III (emission by electron beams) and type IV (trapped electrons) being the relevant ones for SEP events.

Numerous catalogs of proton (and electron) events also identified the radio signatures provided as complementary information to the particle event lists, e.g., Kahler (1982), Cane et al. (2010), Vainio et al. (2013), Richardson et al. (2014), Papaionnou et al. (2016), or radio bursts are used in timing studies between the extrapolated particle injection at the Sun and the release of the radio burst (e.g., Koloumvakos et al. (2015) and the referenced therein) as well as for SEP events forecasting (e.g., Balch et al. 2008; Laurenza et al. 2009; Kahler and Ling 2015; Winter and Ledbetter 2015; Alberti et al. 2017). Listing all works using radio bursts in the studies of SEP events is, however, beyond the scope of this paper.
The present study is based on the statistical results of SEPrelated radio bursts over the period 1997-2006 by Miteva et al. (2013), as well as the analysis covering the period 1996-2016 by Miteva et al. (2017a,b), which can be considered as the revised and extended version of Miteva et al. (2013). The latter studies use a set of criteria on the appearance or not of given radio burst type combination in order to relate to the certainty of the proposed solar origin. In this work, the above line of research will be followed and further explored covering the same time period, namely the previous solar cycle (SC), SC23 (1996-2008), and the ongoing SC24 (2009-2016). Although SC24 is not complete, few events are expected to occur in the final years of the cycle (20172019). Thus, in this study the period of eight years is regarded as representative for the entire SC24.

\section{Event selection}

The event list used here is adopted from Miteva et al. (2017b), in the period 1996-2016. There are 431 proton events listed there, together with their identified radio bursts. The solar origin identification procedure and the radio burst type association, related to the proton-related flares and CMEs, are explained in detail there.

We adopt the criteria for Flare-driven/CME-driven/Mixedcontribution to the particle flux as proposed by Miteva et al. (2017b), according to the different combinations of radio bursts in the specific radio wavelength ranges. However, there are 40 events with no data both in $\mathrm{dm}$ and decimetric-metric (dm-m) ranges that were kept there under the assumption that over the entire sample the event identification will not be significantly modified in case radio data is found. Subsequently, these cases were not excluded which also increased the statistics. Thus, this earlier study should be considered as an upper limit for the event categories described below, in terms of proposed definition and event number. In contrast to the first version of the categorization provided in Miteva et al. (2017b), in this study a more conservative approach is adopted and the events with data gaps in both $\mathrm{dm}$ and $\mathrm{dm}-\mathrm{m}$ range are dropped from further analysis. Nevertheless, events that have data gap either in the dm-range or in the $\mathrm{dm}-\mathrm{m}$ range are kept in the event list.

In summary, there are three main sub-groups proposed in view of the flare vs. CME classification scheme: particles influenced mainly by flares, mainly by CMEs, or by both, as summarized below. Note that this categorization is made only under the assumption of radio burst occurrence without imposing additional restrictions on the particles and their solar origin, flares or CMEs. Thus, it can be regarded as a different approach and an alternative SEP classification scheme, compared to SEP classifications based on in situ particle data. Each sample can be considered as a subset (out of the entire list) containing protons and their associated flares, CMEs and radio bursts. 
The following definition for the SEP-classification is proposed accounting for the physical conditions during the dominant particle acceleration process taking place in the respective sample:

Flare-driven event category: 16\% (71/431); no type II bursts present; type III bursts are present in the dynamic spectrum; type IV bursts can be present in either frequency range.

$>$ certain (13 cases): Type III bursts from $\mathrm{dm}$ or $\mathrm{dm}-\mathrm{m}$ to km-range are identified

$>$ probable (58 cases): Type III bursts from $\mathrm{m}$ to $\mathrm{km}$-range are identified

CME-driven event category: 35\% (149/431); no dm-to-m type III bursts present, type II bursts are present in specific ranges of the dynamic spectrum, as given below; type IV bursts can be present in either frequency range.

$>$ certain (38 cases): type II bursts are identified only in the Dekametric-Hectometic-kilometric (DH) range

$>$ probable (111 cases): type II bursts are identified from low corona $(\mathrm{m})$ to $\mathrm{DH}$ range (no physical continuation is implied)

Mixed-contribution category: $32 \%$ (139/431); dm-m type III bursts and dm-m type II bursts are both present.

Data gaps: 17\% (72/431); data gaps simultaneously in the $\mathrm{dm}$ and $\mathrm{dm}-\mathrm{m}$ range or data gap in the $\mathrm{m}$-to-km ranges; these events are dropped from the statistics and further in this study the remaining 359 events are considered as the entire event sample.

Thus the percentages reported earlier for all event categories (Miteva et al. 2017b), with the exception of the data gaps, are now reduced. Each of the three categories of events contains information on protons, and their solar origin - flares and CMEs. Namely, to each of the above categories there is related sample of protons, flares and CME events, which are investigated in detail below.

\section{Results}

\subsection{Overall distributions}

Out of the entire sample, based on 359 events and given below as a reference value, three main categories of events are distinguished: Flare-driven, CME-driven and Mixed-contribution event samples, respectively. In addition, several time periods are considered: 1996-2016 (denoted as SC23+SC24), 1996-2008 (SC23) and 2009-2016 (denoted as SC24, although the current SC is still ongoing).

The proton intensity is divided, according to the median value of the proton intensity for the entire sample, into large (denoted with L, namely all events with proton intensities larger than the median) and small (denoted with S, namely smaller than the median value). Their number and percentage (as a ratio to the respective sample size) are calculated for each category and given in Table 1. Namely, the Flare-driven category consists of $27 \%$ of larger protons, whereas in the remaining three categories the large protons are nearly equally distributed, from $55 \%$ (for the Mixed category) to $51 \%$ (for the entire sample), respectively. Overall, these rates are similar when performing the same calculations in the time periods over SC23 and SC24 (with the exception of the Mixed category behavior in SC24 when the fraction of large protons is $76 \%$ ).

Additionally, for the same category the percentages of Western (W) and Eastern (E) helio-longitude of the solar origin, evaluated either as the direction of propagation of the CME and/or the longitude of the flare AR, are calculated. The results are close to the value of $70 \%$ for $\mathrm{W}$ and $30 \%$ for E-location (only the Mixed category has slightly larger value of $77 \%$ for events originating from W-location for the entire sample and $85 \%$ for the events in SC24, see Table 1).

Table 1: Table of the occurrence (in percentage) of proton intensity and direction of propagation for the $\mathrm{CME} /$ longitude of the active region (AR) within each event category: Flare-driven, CME-driven, Mixedcontribution and Entire event sample, respectively. The events with data gaps are being dropped. The number of events to the number of events in the entire sub-sample is given in brackets. Abbreviations used: L: large proton events; S: small proton events; W: western longitude of the AR or direction of propagation for the CME, E: eastern; unc: uncertain.

\begin{tabular}{|c|c|c|c|c|}
\hline & $\begin{array}{l}\text { Flare-driven } \\
\text { category }\end{array}$ & $\begin{array}{l}\text { CME-driven } \\
\text { category }\end{array}$ & $\begin{array}{l}\text { Mixed-contribution } \\
\text { category }\end{array}$ & $\begin{array}{l}\text { Entire event } \\
\text { sample }\end{array}$ \\
\hline \multicolumn{5}{|c|}{ SC23+SC24 (1996-2016) } \\
\hline proton intensity & $\begin{array}{l}\text { L: } 27 \%(19 / 71) \\
\text { S: } 73 \%(52 / 71)\end{array}$ & $\begin{array}{l}\text { L: } 53 \%(79 / 149) \\
\text { S: } 47 \%(70 / 149)\end{array}$ & $\begin{array}{l}\text { L: } 55 \%(77 / 139) \\
\text { S: } 45 \%(62 / 139)\end{array}$ & $\begin{array}{l}\text { L: } 49 \%(175 / 359) \\
\text { S: } 51 \%(184 / 359)\end{array}$ \\
\hline $\begin{array}{l}\text { direction of } \\
\text { propagation/ } \\
\text { AR longitude }\end{array}$ & $\begin{array}{l}\text { W: } 71 \%(50 / 71) \\
\text { E: } 28 \%(20 / 71) \\
\text { unc: } 1 \%(1 / 71)\end{array}$ & $\begin{array}{c}\text { W: } 68 \%(102 / 149) \\
\text { E: } 32 \%(47 / 149)\end{array}$ & $\begin{array}{l}\text { W: } 77 \%(107 / 139) \\
\text { E: } 22 \%(31 / 139) \\
\text { unc: } 1 \%(1 / 139)\end{array}$ & $\begin{array}{c}\text { W: } 72 \%(259 / 359) \\
\text { E: } 27 \%(98 / 359)\end{array}$ \\
\hline \multicolumn{5}{|c|}{ SC23 (1996-2008) } \\
\hline proton intensity & $\begin{array}{l}\text { L: } 25 \%(12 / 48) \\
\text { S: } 75 \%(36 / 48)\end{array}$ & $\begin{array}{l}\text { L: } 52 \%(41 / 79) \\
\text { S: } 48 \%(38 / 79)\end{array}$ & $\begin{array}{l}\mathrm{L}: 49 \%(51 / 105) \\
\text { S: } 51 \%(54 / 105)\end{array}$ & $\begin{array}{l}\text { L: } 45 \%(104 / 232) \\
\text { S: } 55 \%(128 / 232)\end{array}$ \\
\hline $\begin{array}{l}\text { direction of } \\
\text { propagation/ } \\
\text { AR longitude }\end{array}$ & $\begin{array}{l}\text { W: 73\% (35/48) } \\
\text { E: } 25 \%(12 / 48) \\
\text { unc: } 2 \%(1 / 48)\end{array}$ & $\begin{array}{l}\text { W: } 68 \%(54 / 79) \\
\text { E: } 32 \%(25 / 79)\end{array}$ & $\begin{array}{l}\text { W: } 74 \%(78 / 105) \\
\text { E: } 25 \%(26 / 105) \\
\text { unc: } 1 \%(1 / 105)\end{array}$ & $\begin{array}{c}\text { W: 72\% (167/232) } \\
\text { E: } 27 \%(63 / 232) \\
\text { unc: } 1 \%(2 / 232)\end{array}$ \\
\hline \multicolumn{5}{|c|}{ SC24 (2009-2016) } \\
\hline proton intensity & $\begin{array}{l}\text { L: } 30 \%(7 / 23) \\
\text { S: } 70 \%(16 / 23)\end{array}$ & $\begin{array}{l}\text { L: } 54 \%(38 / 70) \\
\text { S: } 46 \%(32 / 70)\end{array}$ & $\begin{array}{l}\text { L: } 76 \%(26 / 34) \\
\text { S: } 24 \%(8 / 34)\end{array}$ & $\begin{array}{l}\text { L: } 56 \%(71 / 127) \\
\text { S: } 44 \%(56 / 127)\end{array}$ \\
\hline $\begin{array}{l}\text { direction of } \\
\text { propagation/ } \\
\text { AR longitude }\end{array}$ & $\begin{array}{l}\text { W: } 65 \%(15 / 23) \\
E: 35 \%(8 / 23)\end{array}$ & $\begin{array}{l}\text { W: } 69 \%(48 / 70) \\
\text { E: } 31 \%(22 / 70)\end{array}$ & $\begin{array}{l}\text { W: } 85 \%(29 / 34) \\
\text { E: } 15 \% \quad(5 / 34)\end{array}$ & $\begin{array}{l}W: 72 \%(92 / 127) \\
E: 28 \%(35 / 127)\end{array}$ \\
\hline
\end{tabular}



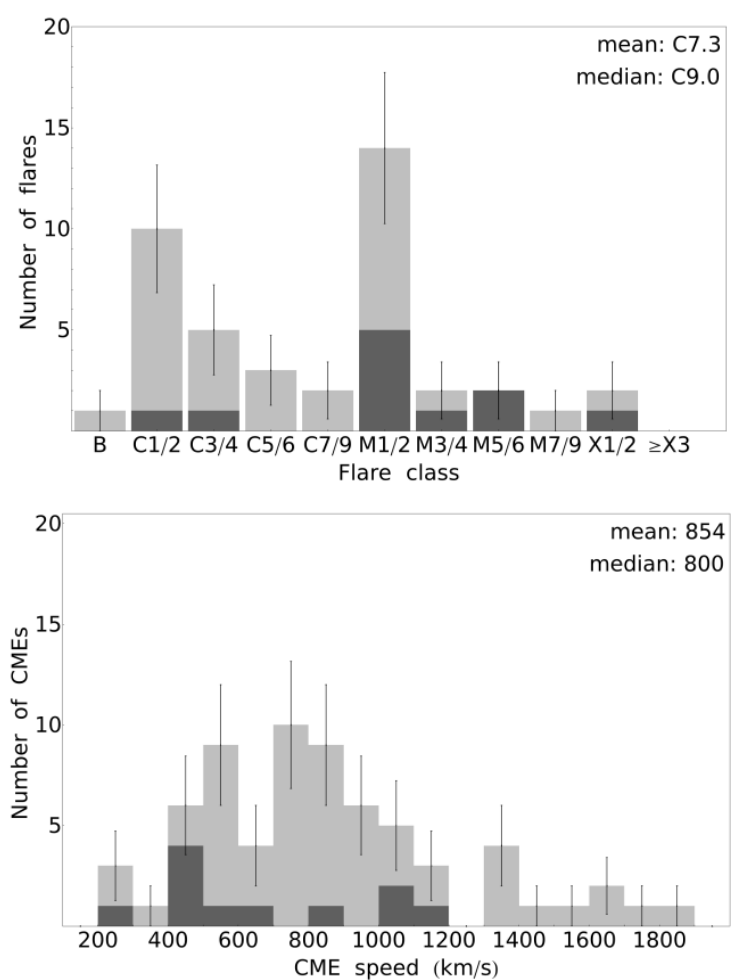
Figure 1: Histograms for the flare class, flare rise time, CME speed and AW for the Flare-driven event category. Dark-gray color
denoted the number of certain cases, light-gray - the probable cases, and the number of events is the length of the colored bar. The mean/median values are shown on each plot. The vertical bars denote the standard error calculated over the entire bin height.

In each of these categories, there are data gaps in associating flares and CMEs to the proton events due to back-sided solar eruptions, complex cases or data gaps. Thus the number of flare and CME sample used for the statistical study below is always less than the proton event sample in the respective category. The exact event numbers are noted while describing the solar origin distributions. Namely, histograms are built for the flare class (in soft-X-ray, SXR, emission), flare (onset-to-peak) rise time, CME projected (reported as linear) speed and the CME angular width (AW).

The distributions are organized as follows. For the flare class, the range $\mathrm{C} 1$-to-X2 is plotted into bins with a width of 1-2(3) flare class units, whereas one bin is reserved for all B-class flares and one bin - for all flares $\geq X 3$. The flare rise time is plotted with a 2min bin width, the CME speed - with a $100 \mathrm{~km} / \mathrm{s}$, whereas the $\mathrm{AW}$ is shown using 25-degree bins.

\subsubsection{Distributions of the Flare-driven category}

Flare-driven sample consists of 71 proton events. For this sample flares could be identified for 42 of the proton cases and CMEs - for 66 cases, which are used for the statistics below. Based on these reduced samples, when the flare characteristics are considered 11 cases (out of 13) are left to be of certain flare origin and the remaining 31 are of probable flare-origin (according to the new SEP categorization proposed above). For the CMEs, there are also 11 certain cases. In Figure 1, upper row are shown the histograms for flare class and flare rise time, and in the lower row - for CME speed and CME AW. The mean/median values are given on each respective plot. The distribution is given as a stacked histogram, namely the colored section (the height of each colored bar) denotes the number of events in each respective group (certain, given with dark-gray and probable origin, given with light-gray color). The sum of the two colored sections gives the total number of events in each bin.
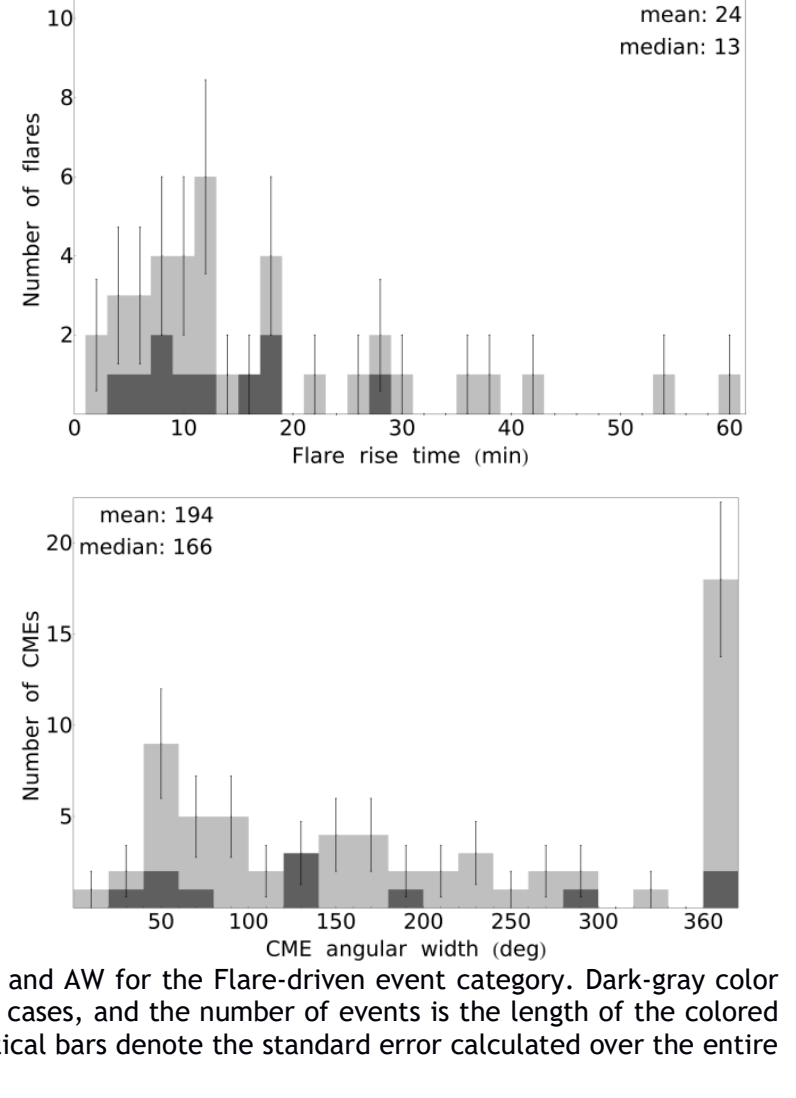

The flare class distribution is double-peaked, where two bins (at $\mathrm{C} 1 / 2$ and M1/2) rising higher than the remaining ones. These bins contain 6 (out of 11) certain cases and are denoted with darkgray color on the plot. The mean/median values (calculated for the entire sample of 42 cases) is C7.3/C9.0, respectively.

The standard error is calculated as a square root of the number of all events in each bin (the latter is denoted by the bin height). Although the standard error is relatively large, the described trends are evident. For clarity purposes, in Fig. 1 the standard error is calculated over the entire bin, and not only on the light-colored section. The standard error for the certain cases (dark-colored bins) will have the same value as the already depicted error bar on a neighboring bin (of any color combination) of the same height.

Flare rise time (with individual values for the different cases ranging from 3 to $162 \mathrm{~min}$ ) is shown over a period of one hour for clarity purpose; however the mean/median values (24/13 $\mathrm{min})$ are calculated over the entire event sample. The majority of the cases have a flare rise time within about $20 \mathrm{~min}$, including the majority of the certain cases; one is not shown since it has a value of 80 min. The obtained values for the flare rise time for this category are consistent with the properties of 'impulsive flares' proposed by of Park et al. (2010), however the flare-driven sample defined here is not CME-free, namely by definition it does not exclude the presence or indirect influence of CMEs on the particle flux.

Thus, as the other categories, the flare-driven (or flaredominated) one contains information on proton events and their solar origin - flares and CMEs. Namely, in the flare-driven category CME properties can be obtained in terms of linear speed and AW. The mean/median values for the CME speed are $854 / 800 \mathrm{~km} / \mathrm{s}$ respectively, with a broad distribution $200-1800$ $\mathrm{km} / \mathrm{s}$. The certain cases cluster over 400-600 and 1000-1100 km/s bins. With respect to the CME AW, the flare-driven category consists of rather narrow CMEs with mean/median values of 194/166 degrees, respectively, without a clear preference for the AW of the certain cases. 

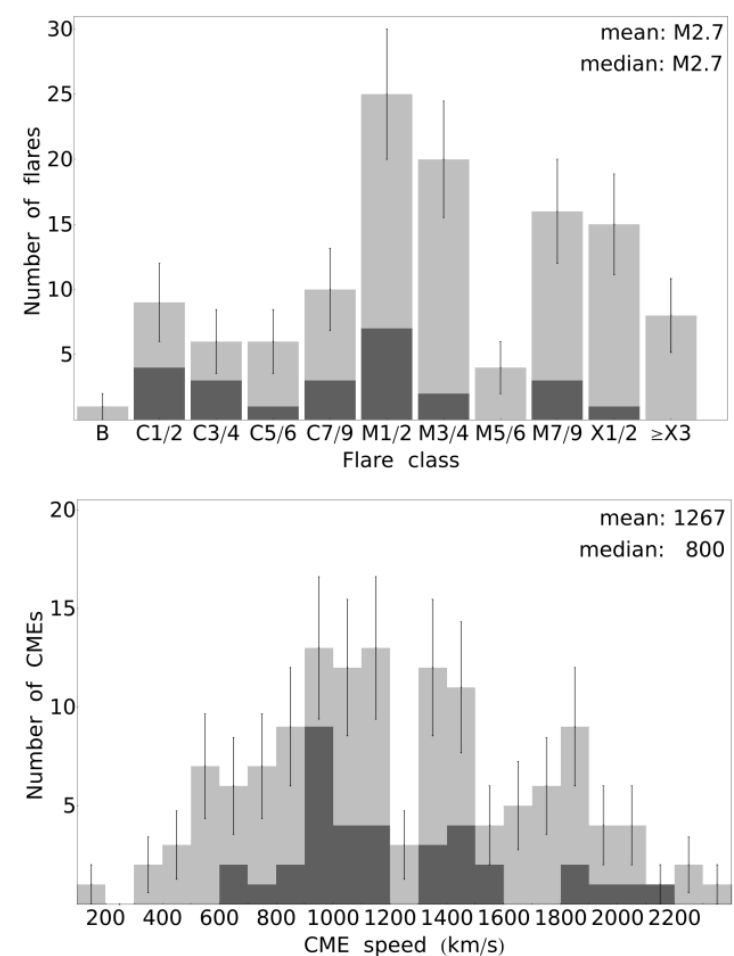

Figure 2: Distribution $\mathrm{CME}$ speed $(\mathrm{km} / \mathrm{s})$ error bars as in Fig. 1.

\subsubsection{Distributions of the CME-driven category}

For the CME-driven category (149 proton events), flare association was possible for 120 cases and CME association - for 140 cases. The certain category consists of 38 events.

Similarly as discussed above, the CME-driven category contains also flare information (in addition to the protons and radio bursts). The flare class distribution for the CME-driven category peaks also at two places: first at M1/2 but also at a higher flare class, M7/9, compared to the Flare-driven category. The mean/median value is M2.7, and the certain cases show no clear preferential distribution. For this category, the flare rise time is longer, $36 / 23 \mathrm{~min}$ for the mean/median value, respectively (some certain cases have rise times longer than $60 \mathrm{~min}$ ).

With respect to the CME speed, the median value is the same $(800 \mathrm{~km} / \mathrm{s})$ as for the flare-driven category, with larger value for the mean speed $(1267 \mathrm{~km} / \mathrm{s})$ with much broader distribution $(100-$ $2400 \mathrm{~km} / \mathrm{s}$ ). The certain cases cluster around 800-1100 km/s. With respect to the AW, the CMEs are mostly halo, with mean/median value of $306 / 360$ degrees, respectively, with the largest fraction of the certain cases being halo CMEs.

\subsubsection{Distributions of the Mixed-contribution category}

The Mixed-contribution category consists of 139 proton events, with flare identification for 132 cases and CME identification for 129 cases.

The histogram for the flare class for this category is unlike the other two categories because it is nearly flat in the range
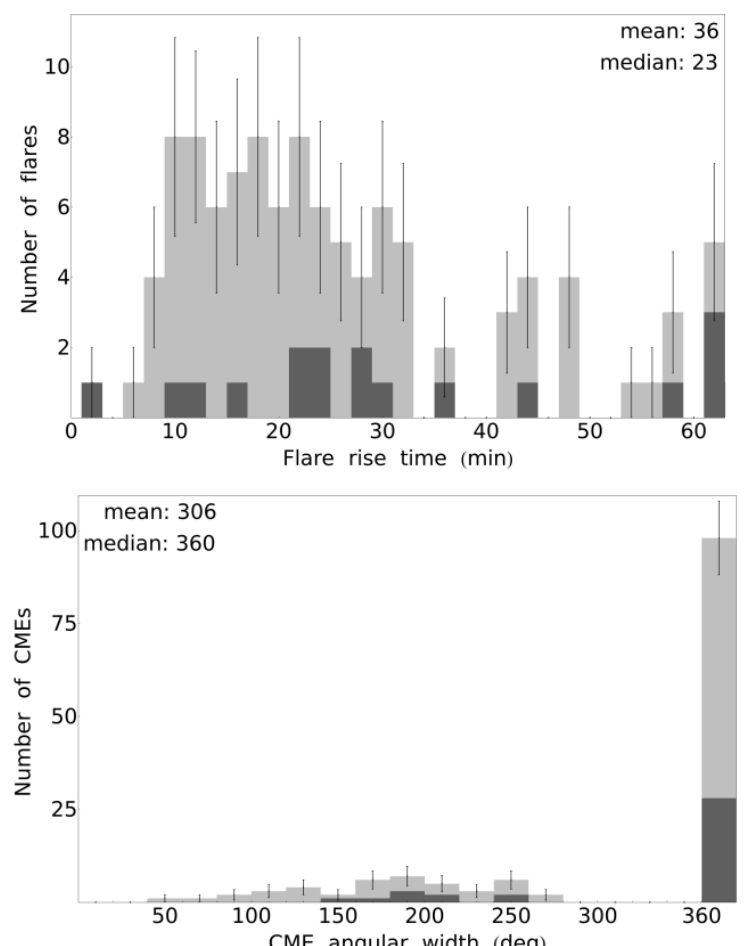

$M 1 / 2$ to $\geq X 3$, with a slightly higher bin for M1/2. The mean/median values are about M5. The flare rise time distribution has a longer tail towards larger values. Many flares with a gradual increase comprise this category, although the mean/median values are $25 / 20 \mathrm{~min}$, respectively, which is close to the mean value of the Flare-driven category.

The median value for the CME speed is the largest, $1100 \mathrm{~km} / \mathrm{s}$, with nearly flat distribution between 500 and 1500 $\mathrm{km} / \mathrm{s}$ and extending to over $3000 \mathrm{~km} / \mathrm{s}$. The mean value of 1200 $\mathrm{km} / \mathrm{s}$ is close to the mean value for the CME-driven category. The CMEs are halo, with values of $277 / 360$ degrees for the mean/median AW, respectively, both reminiscent to the values of the CME-driven category.

\subsubsection{Solar cycle trends}

The above distributions consider the entire time period (19962016). In this section, the SC behavior of the flare and CME mean/median values are investigated. The results are summarized in Table 2.

For the flare and CME-driven categories the mean/median values for the flare class and CME speed are larger in SC23 compared to the respective values for the entire sample (SC23+SC24). Alternatively, these values are smaller in SC24. Overall, the differences are within the standard error. The values for the flare rise time and CME AW, however, do not show clear SC change. The flare and CME characteristics for the Mixedcontribution category neither show strong SC dependence. 

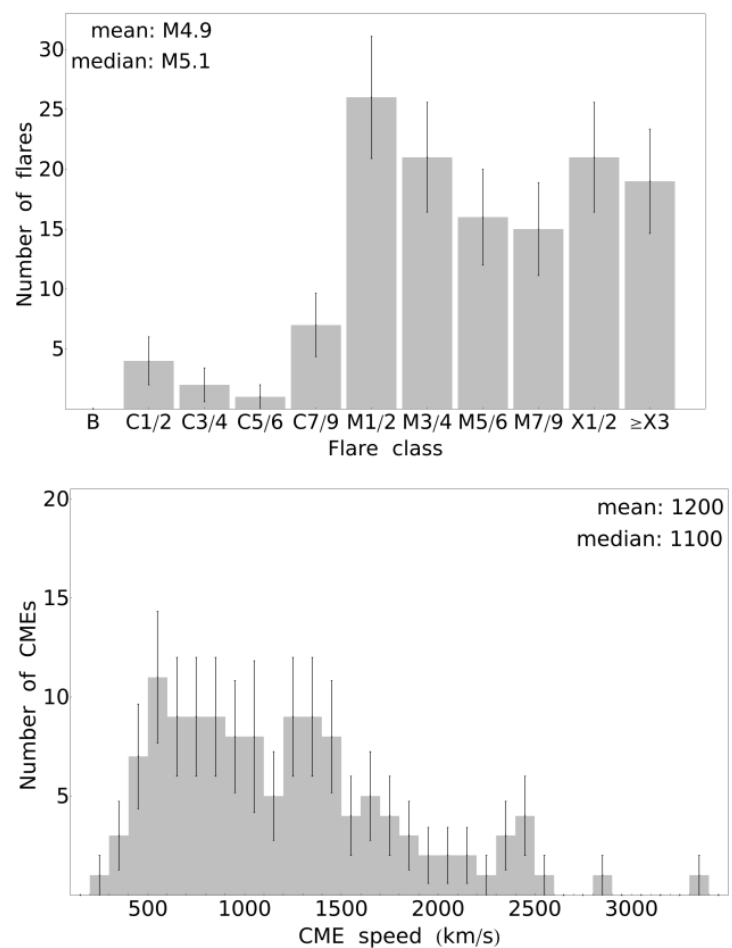

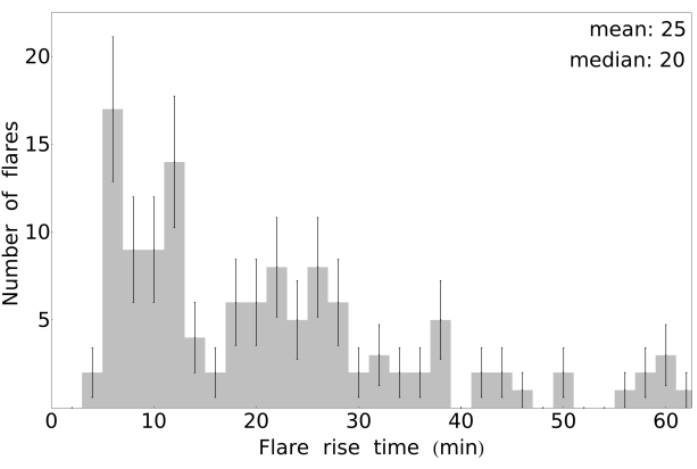

mean: 277

75 median: 360

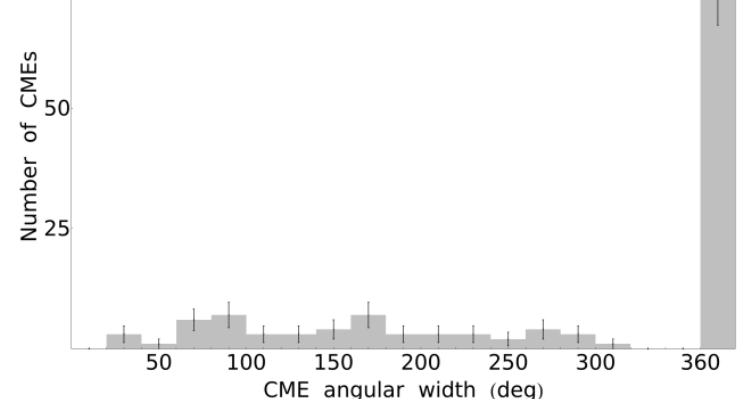

Figure 3: Distribution of the flare class, flare rise time, CME speed and AW for the Mixed-contribution event category. The length of the color bar denotes the event number in the respective bin. Error bars as in Fig. 1.

Table 2: Table of the mean/median values of the flare class, flare rise time (in $\mathrm{min}$ ), CME speed (in $\mathrm{km} / \mathrm{s}$ ) and AW (in deg) in the different event categories and time periods, respectively. In brackets is given the number of events in each case over which the mean/median is calculated.

\begin{tabular}{|l|c|c|c|}
\hline \multicolumn{5}{|c|}{$\begin{array}{c}\text { Flare-driven } \\
\text { category }\end{array}$} & $\begin{array}{c}\text { CME-driven } \\
\text { category }\end{array}$ & $\begin{array}{c}\text { Mixed-contribution } \\
\text { category }\end{array}$ \\
\hline Flare class & C7.3/C9.0 (42) & M2.7/M2.7 (120) & M4.9/M5.1 (132) \\
\hline Flare rise time & $24 / 13(42)$ & $36 / 23(119)$ & $25 / 20(132)$ \\
\hline CME speed & $854 / 800(66)$ & $1267 / 800(140)$ & $1200 / 1100(129)$ \\
\hline CME AW & $194 / 166(66)$ & $306 / 360(140)$ & $277 / 360(129)$ \\
\hline \multicolumn{5}{|c|}{ SC23 (1996-2008) } \\
\hline Flare class & C8.0/C9.5 (33) & M3.6/M3.7 (69) & $M 4.7 / M 5.1(102)$ \\
\hline Flare rise time & $23 / 12(33)$ & $33 / 22(69)$ & $23 / 19(102)$ \\
\hline CME speed & $908 / 851(43)$ & $1364 / 1336(70)$ & $1197 / 1090(95)$ \\
\hline CME AW & $181 / 152(43)$ & $294 / 360(70)$ & $261 / 360(95)$ \\
\hline \multicolumn{5}{|c|}{ SC24 } & $\mathbf{2 0 0 9 - 2 0 1 6 )}$ \\
\hline Flare class & C5.4/C3.0(9) & M1.8/M2.0 (51) & $M 5.7 / M 5.2(30)$ \\
\hline Flare rise time & $29 / 21(9)$ & $40 / 25(50)$ & $29 / 24(30)$ \\
\hline CME speed & $754 / 753(23)$ & $1170 / 1050(70)$ & $1200 / 1204(34)$ \\
\hline CME AW & $220 / 227(23)$ & $319 / 360(70)$ & $322 / 360(34)$ \\
\hline
\end{tabular}

\subsection{Statistical differences}

Here, the statistical difference between pairs of the above distributions is evaluated based on the entire time period. The statistical significance, $D$, between two samples of different size is tested using the two-sample Kolmogorov-Smirnov test. Namely, two samples of the same phenomena, either flare class/rise time or CME speed/AW, are evaluated if they are statistically different. For this study, a significance level of $\alpha=0.05$ is adopted (giving for the coefficient $c(0.05)=1.36$ ). Depending on the number of events in either sample, a critical value is calculated as: $D_{0.05}=1.36\left[\left(n_{1}+n_{2}\right) / n_{1} n_{2}\right]^{0.5}$, where $n_{1}$ and $n_{2}$ are the number of events in samples 1 and 2, respectively. Statistical significance is fulfilled when $D>D_{0.05}$. The results using the above statistical procedure are summarized in Table 3 . Note that since the event number in the compared samples for flare class and flare rise is similar or the same for CME speed and AW, space respectively, the value for $\mathrm{D}_{0.05}$ is the same.

When the flare sample is considered, using the values for the flare class and flare rise time, the significance is tested for three combinations of samples, in either category: Flare, CME or Mixed. With respect to the flare class and flare rise time, a statistical difference is obtained in the majority of the cases, namely when comparing the samples in each respective pair of categories, including, although just marginally, for the CME and Mixed category, but excluding Flare vs. Mixed categories.

When the CME samples are compared, by using the values for the CME speed and AW, statistical difference is obtained for the categories Flare with CME and Flare with Mixed. The CME and Mixed categories are just marginally the same (if a larger value for $\alpha$ is selected, the samples will fulfill the criteria for difference). 
Table 3: Contingency table for the statistical significance (Yes/No) based on two-sample Kolmogorov-Smirnov test using flare class/rise time and CME speed/AW from the Flare-driven, CME-driven or Mixed-contribution category. For abbreviation see text.

\begin{tabular}{c|c|c|c}
\hline & $\begin{array}{c}\text { Flare-driven vs. } \\
\text { CME-driven }\end{array}$ & $\begin{array}{c}\text { CME-driven vs. } \\
\text { Mixed-contribution }\end{array}$ & $\begin{array}{c}\text { Flare-driven vs. } \\
\text { Mixed-contribution }\end{array}$ \\
\hline Flare class & $\begin{array}{c}\text { Yes } \\
\left(D=0.38 ; D_{0.05}=0.24 ;\right. \\
\left.n_{1}=42 ; n_{2}=120\right)\end{array}$ & $\begin{array}{c}\text { Yes } \\
\left(D=0.19 ; D_{0.05}=0.17 ;\right. \\
\left.n_{1}=120 ; n_{2}=132\right)\end{array}$ & $\begin{array}{c}\text { Yes } \\
\left(D=0.55 ; D_{0.05}=0.24 ;\right. \\
\left.n_{1}=42 ; n_{2}=132\right)\end{array}$ \\
\hline Flare rise time & Yes & Yes & No \\
& $\left(D=0.35 ; D_{0.05}=0.24 ;\right.$ & $\left(D=0.22 ; D_{0.05}=0.17 ;\right.$ & $\left(D=0.20 ; D_{0.05}=0.24 ;\right.$ \\
& $\left.n_{1}=42 ; n_{2}=119\right)$ & $\left.n_{1}=119 ; n_{2}=132\right)$ & $\left.n_{1}=42 ; n_{2}=132\right)$ \\
\hline CME speed & Yes & No & Yes \\
& $\left(D=0.43 ; D_{0.05}=0.20 ;\right.$ & $\left(D=0.15 ; D_{0.05}=0.17 ;\right.$ & $\left(D=0.33 ; D_{0.05}=0.21 ;\right.$ \\
& $\left.n_{1}=66 ; n_{2}=140\right)$ & $\left.n_{1}=140 ; n_{2}=129\right)$ & $\left.n_{1}=66 ; n_{2}=129\right)$ \\
\hline CME AW & Yes & No & Yes \\
& $\left(D=0.43 ; D_{0.05}=0.20 ;\right.$ & $\left(D=0.14 ; D_{0.05}=0.17 ;\right.$ & $\left(D=0.33 ; D_{0.05}=0.21 ;\right.$ \\
& $\left.n_{1}=66 ; n_{2}=140\right)$ & $\left.n_{1}=140 ; n_{2}=129\right)$ & $\left.n_{1}=66 ; n_{2}=129\right)$ \\
\hline
\end{tabular}

\section{Discussion and conclusions}

The present SEP study starts with the radio burst identifications based on about $20 \mathrm{MeV}$ proton event list (Miteva et al. 2017b, see also the correlation analysis presented there). Thus, all results reported here correspond to a proton sample of this energy range. Energy dependent correlation trends have been investigated elsewhere, e.g. Dierckxsens et al. (2015) and Miteva et al. (2017a).

The statistical difference of the flare and/or CME sample distributions in the different SEP-categories is investigated in the present study by means of the two-sample Kolmogorov-Smirnov test. For the majority of the category combinations it is found that the flare/CME sample stem from different distributions and the exceptions are just marginally the same.

The Flare-driven category is the statistically different one from the CME-driven category in all flare and CME properties.

The Mixed-contribution category has a flare class distribution that is statistically different compared to the Flare-driven and CME-driven category, whereas with respect to the flare rise time it is different only from the CME-driven one. The CME distribution of the Mixed category is the same within the $5 \%$ confidence level with the CMEs in the CME-driven sample, but different from the CMEs in the Flare-driven category.

Using findings on the flare and CME distributions in each SEP category it could be argued that the dominant driver of the SEP events is due to differences in the populations of flares and CMEs when formed in the solar corona. Based on radio emission signatures, flare-acceleration of SEPs is possible even from small in terms of their SXR emission and impulsive, with median rise time of about $13 \mathrm{~min}$, solar flares when open magnetic field line configuration from the low to high solar atmosphere is present. This is the case for about $16 \%$ of the entire proton sample. In these cases, the accompanied CMEs are rather slow and narrow and it is not straightforward to expect that they will be sufficient particle accelerators (also type II bursts are absent here). However in this category, smaller in intensity (than median value) proton events are the majority (more than $70 \%$ of the observed proton sample). In summary, the Flare-driven category can include non-thermal protons, however of smaller peak intensity.

In contrast, the CME-dominant acceleration is expected when CMEs faster than the local Alfven speed are formed (e.g., Klein and Trottet, 2001), although the median value for the CME speed in the CME-driven sample is the same as in the Flare-driven category. In addition, halo-CMEs have the potential to be geoeffective (Ameri and Valtonen, 2017). Interestingly, the flares accompanying these CMEs are of larger flare class (M2.7) but are more gradually rising (median value of $23 \mathrm{~min}$ ), compared to the Flare-category.

The Mixed-contribution category contains the most energetic flares and CMEs according to the median values of their distributions. This SEP category is similar to the CME-driven one in terms of CME sample characteristics, e.g., there is no statistically significant difference, and just marginally different in terms of the flare parameters. Larger (about M5) and gradual (rise time of $20 \mathrm{~min}$ ) flares are present in the Mixed-category which are statistically different sample, in terms of flare class, compared to the flare samples in the other two SEP-categories. The CMEs in the Mixed-category are faster (median value of $1100 \mathrm{~km} / \mathrm{s}$ ) compared to the other two categories.

The SC dependence is tested for the large vs. small in intensity protons as well as for the helio-longitudinal location of the flares and CMEs. Over the entire period less than one third of the proton events for the Flare-driven category are of small intensity, compared to $50-55 \%$ for the other categories. With respect to the longitude, the Mixed-contribution category has slightly more western-origin events (77\%) compared to nearly $70 \%$ for the other categories.

These trends are kept in SC23. For the case of SC24, the Mixed category is over-represented with large in intensity protons $(76 \%)$. It also shows more events at western longitudes (85\%, compared to SC23 or the entire time period). In SC24, the Flaredriven and CME-driven categories show western events that are just slightly below $70 \%$.

In the present analysis, the different SEP-categories are defined solely based on radio burst type occurrence. This is an alternative categorization, compared to the electron to proton ratio adopted by others, e.g., Cliver (1996), Cane at al. (2010). This ratio was not considered here as additional parameter. In Cane at al. (2010) was shown that there is a gradual transition between the different SEP categories in contrast to the originally proposed bimodal (impulsive vs. gradual) distribution.

Similarly to the plateau-like distributions proposed by Cane et al. (2010), each of the SEP-related solar flares and CMEs considered in the present study comprise of three distributions with some overlap between them. This triple distribution contains the pool of solar events which finally accelerates the in situ observed particle events. A probable scenario for the SEP origin is a combination of, on one side, the existence of suitable physical conditions for particle acceleration and emission at radio wavelengths, together with, on another side, a specific magnetic field line configuration with a ready access for particle escape via open field lines. Since no timing of the radio burst types was considered at present in the different SEP categories, no conclusion can be made on the release time of the different burst 
types with respect to the flare or CME evolution. In addition, the longitudinal effect is not clearly distinguished, since over the entire sample all categories follow roughly the $70 \%$ vs. $30 \%$ $( \pm 10 \%)$ separation of western vs. eastern location, respectively. It was obtained that shock-free conditions, weaker solar flares and dm-to-km type III bursts can also be a SEP-productive scenario. Thus, the magnetic field configuration (open field lines from the very low corona) could play a greater role than previously expected in the methodology of solar origin identification (as discussed by Klein et al. 2008), since small and impulsive flares could lead to a population of large intensity proton events of about $20 \mathrm{MeV}$.

\section{Acknowledgements}

This study is supported by the National Science Fund of Bulgaria, contract No. DNTS/Russia 01/6 (23-Jun-2017), under the Project of collaborative research between Bulgaria and Russia 'The origin of solar energetic particles: solar flares vs. coronal mass ejections'.

\section{References}

Alberti, T., Laurenza, M., Cliver, E. W. et al.: 2017, ApJ, 838, 59, 11 pp. doi: $10.3847 / 1538-4357 /$ aa5cb8

Ameri, D., Valtonen, E.: 2017, Solar Physics, 292, 79, 20 pp. doi: 10.1007/s11207-017-1102-7

Balch, C. C.: 2008, Space Weather, 6, S01001, 13 pp. doi: 10.1029/2007SW000337

Cane, H. V., Richardson, I. G., von Rosenvinge, T. T.: 2010, JGR, 115, A8, A08101. doi: 10.1029/2009JA014848

Cliver, E.: 2009, Central European Astrophysical Bulletin, 33, pp. 253-270.

Cliver, E.: 1996, AIP Conference Proceedings, 374, pp. 45-60. doi: $10.1063 / 1.50980$

Dierckxsens, M.; Tziotziou, K.; Dalla, S. et al.: 2015, Solar Physics, 290, pp. 841-874. doi: 10.1007/s11207-014-0641-4

Kahler, S. W.: 1982, ApJ, 261, pp. 710-719. doi: 10.1086/160381

Kahler, S. W., Ling, A.: 2015, Space Weather, 13, pp. 665-675. doi: $10.1002 / 2015 S W 001222$

Kallenrode, M.-B.; Wibberenz, G.: 1991, ApJ, 376, pp. 787-796. doi: $10.1086 / 170327$

Klein, K.-L., Krucker, S., Lointier, G., Kerdraon, A.: 2008, A\&A, 486, pp. 589-596. doi: 10.1051/0004-6361:20079228

Klein, K.-L., Trottet, G.: 2001, Space Science Reviews, 95, pp. 215-225.

Krucker, S., Lin, R. P.: 2000, ApJ, 542, pp. L61-L64. doi: $10.1086 / 312922$

Kouloumvakos, A., Nindos, A., Valtonen, E. et al.: 2015, A\&A, 580, A80, 17 pp. doi: 10.1051/0004-6361/201424397
Laurenza, M., Cliver, E. W., Hewitt, J. et al.: 2009, Space weather, 7, S04008, 18 pp. doi: 10.1029/2007SW000379

Lin, R. P.: 1970, Solar Physics, 12, pp. 266-303. doi: 10.1007/BF00227122

Miteva, R., Klein, K.-L., Samwel, S. W. et al.: 2013, Central European Astrophysical Bulletin,, 37, pp. 541-553.

Miteva, R.; Samwel, S. W.; Krupar, V.: 2017a, Proceedings of the $9^{\text {th }}$ Workshop 'Solar Influences on the Magnetosphere, Ionosphere and Atmosphere', edited by K. Georgieva, B. Kirov and D. Danov, ISSN 2367-7570, pp. 19-23.

Miteva, R., Samwel, S. W., Krupar, V.: 2017b, JSWSC, 7, A37, 15 pp. doi: 10.1051/swsc/2017035

Nindos, A.; Aurass, H.; Klein, K.-L.; Trottet, G.: 2008, Solar Physics, 253, pp. 3-41. doi: 10.1007/s11207-008-9258-9

Papaioannou, A., Sandberg, I., Anastasiadis, A. et al.: 2016, JSWSC, 6, A42, 29 pp. doi:

Park, J., Moon, Y.-J., Lee, D. H., Youn, S.: 2010, JGR, 115, A10105, 6 pp. doi: 10.1029/2010JA015330

Pick, M. and Vilmer, N.: 2008, Astronomy and Astrophysics Reviews, 16, pp. 1-153. doi: 10.1007/s00159-008-0013-x

Reames, D. V.: 1999, Space Science Reviews, 90, pp. 413-491. doi: 10.1023/A:1005105831781

Reames, D. V.: 2013, Space Science Reviews, 175, pp. 53-92. doi: 10.1007/s11214-013-9958-9

Richardson, I. G., von Rosenvinge, T. T., Cane, H. V. et al.: 2014, Solar Physics, 289, pp. 3059-3107. doi: 10.1007/s11207-0140524-8

Vainio, R., Valtonen, E., Heber, B., et al.: 2013, JSWSC, 3, A12, 17 pp. doi: $10.1051 /$ swsc/2013030

Winter, L. M., Ledbetter, K.: 2015, ApJ, 809, 105, 19 pp. doi: $10.1088 / 0004-637 X / 809 / 1 / 105$ 